\title{
Susceptibilidad a antibióticos en cepas de Pseudomonas aeruginosa aisladas de canes con otitis externa
}

\author{
Luján, D.A. 1,2; Saavedra, I. ${ }^{2}$; Luján, L.M. ${ }^{2}$ \\ ${ }^{1}$ Postgrado en Infectología y Med. Trop. Facultad de Medicina, Univ.Fed.Minas Gerais, \\ Belo Horizonte, Brasil. ${ }^{2}$ Clínica Vet. La Colonial, Callao, Perú. \\ E-mail: daniellujanroca@gmail.com
}

\begin{abstract}
Resumen
Luján, D.A.; Saavedra, I.; Luján, L.M.: Susceptibilidad a antibióticos en cepas de Pseudomonas aeruginosa aisladas de canes con otitis externa. Rev. Vet. 31: 1, 82-84, 2020. El propósito de este estudio fue determinar la susceptibilidad a los antibióticos en cepas de $P$. aeruginosa aisladas de canes con otitis externa en Callao, Perú. Veinte cepas fueron probadas en su susceptibilidad a 11 antibióticos mediante el método de disco difusión. Los aminoglucósidos presentaron la mejor sensibilidad $(94,4 \%)$. La multirresistencia se observó en un $70 \%$. Los animales de raza definida fueron los más afectados $(50 \%)$. Los aminoglucósidos y seguidamente las fluoroquinolonas se mantienen como opciones de tratamiento.
\end{abstract}

Palabras clave: perros, otitis externa, Pseudomonas aeruginosa, antibióticos.

\begin{abstract}
Luján, D.A.; Saavedra, I.; Luján, L.M.: Antibiotic susceptibility of Pseudomonas aeruginosa strains isolated from dogs with external otitis. Rev. Vet. 31: 1, 82-84, 2020. The purpose of this study was to determine susceptibility to antibiotics in strains of $P$. aeruginosa isolated from dogs with external otitis in Callao, Perú. Twenty strains were tested for their susceptibility to 11 antibiotics by the disk diffusion method. The aminoglycosides presented the best sensitivity (94.4\%). Multiresistance was registered in 70\%. Mongrel breed were the most affected (50\%). The aminoglycosides and then the fluoroquinolones remain as treatment options.
\end{abstract}

Key words: dogs, external otitis, Pseudomonas aeruginosa, antibiotics.

\section{INTRODUCCIÓN}

Una de las principales patologías que afectan a los perros de cualquier raza o género es la otitis externa $(\mathrm{OE})$, que ocasiona prurito intenso, dolor, otorrea persistente uni o bilateral, así como olor fétido ${ }^{18}$. Se encuentra incluida entre los problemas dermatológicos, alcanzando una frecuencia de $10-20 \%$ entre la población canina ${ }^{5,18}$. Muchos microorganismos pueden ocasionarla, entre ellos, Pseudomonas sp, Staphylococcus sp, Streptococcus sp y Escherichia coli ${ }^{4,13,27}$.

Pseudomonas aeruginosa, bacteria Gram negativa, aerobia, no fermentadora de la glucosa, es una de las predominantes en la OE (Rev.Biomed. 19: 156-160; Acta Bioq.Clin.Lat. 48: 465-474), indicándose su presencia hasta en un $11,9 \%{ }^{24}$, siendo variable su susceptibilidad a los antibióticos, lo cual se agrava debido a los múltiples mecanismos de resistencia que presenta ${ }^{20,23}$.

El propósito de este estudio fue constatar los niveles de susceptibilidad a los antibióticos comúnmente usados en la OE en una clínica veterinaria privada durante un período de tres años.

Recibido: setiembre 2019 / Aceptado: enero 2020

\section{MATERIAL Y MÉTODOS}

La vigilancia prospectiva se realizó desde una clínica veterinaria ubicada en el distrito de Bellavista, Callao, Perú. Se incluyeron los resultados de los pacientes con sospecha de otitis, a los que se les solicitó un análisis de laboratorio entre enero de 2013 y diciembre de 2015.

A cada paciente se le efectuó un aislamiento con o sin tratamiento previo con antibióticos. La muestra fue tomada del canal auditivo externo, sembrada en agar McConkey y agar sangre, incubada por $24 \mathrm{~h}$ a $37^{\circ} \mathrm{C}$, e identificada en base a sus características de cultivo, morfológicas y bioquímicas (utilización de lactosa, glucosa, citrato, prueba de oxidasa y crecimiento a $42^{\circ} \mathrm{C}$ ).

Los análisis se realizaron en el Laboratorio de Microbiología y Parasitología de la Facultad de Medicina Veterinaria de la Universidad Nacional Mayor de San Marcos (Lima, Perú). La susceptibilidad a los antibióticos fue evaluada con el método de difusión en agar (disco de Bauer-Kirby), acorde a las recomendaciones del Clinical and Laboratory Standards Institute ${ }^{6}$. Las cepas con susceptibilidad intermedia se consideraron sensibles. 
El antibiograma incluyó varias clases de antibióticos, a saber: 1: betalactámicos: [penicilinas], amoxicilina/ácido clavulánico $(30 \mu \mathrm{g})$ [cefalosporinas], cefalexina $(30 \mu \mathrm{g})$, ceftriaxona $(30 \mu \mathrm{g})$. 2: fluoroquinolonas: ciprofloxacina $(5 \mu \mathrm{g})$, enrofloxacina $(5 \mu \mathrm{g})$. 3 : aminoglucósidos: gentamicina $(10 \mu \mathrm{g})$, amikacina $(30 \mu \mathrm{g})$, neomicina $(30 \mu \mathrm{g})$, tobramicina $(10 \mu \mathrm{g}$,). 4: lincosaminas: clindamicina $(2 \mu \mathrm{g})$. 5: sulfamidas: sulfametoxazoltrimetoprima $(25 \mu \mathrm{g})$. La multirresistencia se definió como resistencia a tres o más clases de antibióticos ${ }^{25}$.

El análisis estadístico se efectuó con la versión 3.03 del programa OpenEpi, determinándose proporciones simples, intervalos de confianza y prueba chi-cuadrado $(\chi 2)$.

\section{RESULTADOS Y DISCUSIÓN}

De un total de 65 cultivos bacterianos, 20 cepas de P. aeruginosa $(30,8 \%$, IC $95 \%=20,48-42,74)$ fueron aisladas en el período de estudio. Se registró una población heterogénea representada por 11 machos $(55 \%$, IC $95 \%=33,28-75,36)$ y 9 hembras ( $45 \%$ IC $95 \%=24,64$ - 66,72). No hubo diferencias de infección según el sexo $(\mathrm{p}<0,05)$. El promedio de edad fue de cinco años y dos meses. La frecuencia de las cepas fue de 7, 4 y 9 en 2013, 2014 y 2015, respectivamente.

La OE es muy común entre las patologías otológicas, afectando principalmente a los canes. Existen diversos factores predisponentes y perpetuantes involucrados, entre estos, las infecciones bacterianas son un ejemplo, teniendo a la $P$. aeruginosa como una de las más frecuentes y difíciles de tratar, debido a su amplia resistencia a los antibióticos ${ }^{1,14}$.

En nuestro estudio la sensibilidad a los aminoglucósidos fue de 94,4\% (34/36) (Tabla 1). Otros estudios obtuvieron registros similares: en el caso específico de gentamicina, ha sido reportada al $98 \%$ en Bulgaria ${ }^{21}$, $94 \%$ en Alemania ${ }^{30}, 85 \%$ en Canada ${ }^{11}, 84,6 \%$ en Dinamarca ${ }^{19}$ y $81 \%$ en Turquía ${ }^{29}$. Porcentajes menores se registraron al 71,4\% en Brasil ${ }^{20}, 70 \%$ en Colombia $^{3}$ y $67 \%$ en Kosovo ${ }^{24}$. En contraste, moderada resistencia fue reportada en Rumania $(43,75 \%){ }^{9}$ y Croacia $(43,5 \%)^{16}$.

Tabla 1. Resistencia a los antibióticos en $P$. aerugino$s a$ aisladas de canes.

\begin{tabular}{lcc}
\hline antibiótico & $\mathrm{N}^{\mathrm{O}}$ & $\%$ \\
\hline amoxicilina/ác.clavulánico & $14 / 18$ & 77,8 \\
cefalexina & $16 / 18$ & 88,9 \\
ceftriaxona & $0 / 4$ & 0 \\
ciprofloxacina & $2 / 20$ & 10 \\
enrofloxacina & $2 / 18$ & 11,1 \\
gentamicina & $0 / 14$ & 0 \\
amicacina & $0 / 4$ & 0 \\
neomicina & $0 / 12$ & 0 \\
tobramicina & $2 / 6$ & 33,3 \\
clindamicina & $6 / 8$ & 75 \\
sulfametoxazol-trimetoprima & $16 / 20$ & 80 \\
\hline
\end{tabular}

Los principales mecanismos de resistencia a los aminoglucósidos en $P$. aeruginosa son la inactivación por enzimas modificantes, las alteraciones en la permeabilidad y la eliminación por bombas de expulsión. Las enzimas más frecuentes son la nucleotidiltransferasa [ANT(2")-I] que confiere resistencia a gentamicina, tobramicina, dibekacina y kanamicina; y la acetiltransferasa [AAC(6')-II] cuyo sustrato es gentamicina, tobramicina y netilmicina ${ }^{17,22}$.

En cuanto a otros porcentajes de resistencia, se registraron valores elevados para cefalexina $(88,9 \%)$, sulfametoxazol-trimetoprima (80\%), amoxicilina/ácido clavulánico $(77,8 \%)$ y clindamicina $(75 \%)$ (Tabla 1). Diversos estudios han determinado porcentajes que

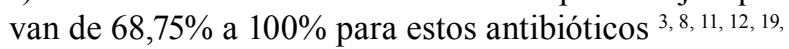
21, 28, 29. Estos valores hallados refuerzan la necesidad del uso racional de los antibióticos en la práctica terapéutica de los casos de $\mathrm{OE}$ basados en pruebas in vitro de susceptibilidad, con el fin de incrementar la efectividad del tratamiento.

Considerando las clases de antibióticos, los mayores porcentajes de resistencia se observaron en el grupo de las sulfamidas $80 \%(16 / 20)$, seguido de los betalactámicos 75\% (30/40) y las lincosaminas 75\% (6/8). Este hecho concuerda con datos obtenidos por otros investigadores ${ }^{2,15,26}$, quienes en cada clase casi siempre probaron un número diferente de antibióticos, los cuales también podían pertenecer a diferentes grupos dentro de una misma clase.

En cuanto a la multirresistencia, el $70 \%$ de las cepas (14/20) presentaron esta característica, notándose que entre ellas, 10 cepas mostraron resistencia a tres clases de antibióticos, 2 frente a cuatro clases y otras 2 frente a cinco clases. Porcentajes menores han sido reportados por otros autores ${ }^{7,26}$.

Este fenómeno involucra un gran desafío actual, ya que la resistencia es seleccionada por el uso de los antibióticos ${ }^{29}$, estando directamente relacionada con el "arsenal genético" a disposición de las bacterias para adquirir e intercambiar genes de resistencia ${ }^{10}$.

Las principales razas afectadas (Tabla 2) fueron: schnauzer (4), shihtzu (2), labrador retriever (2) e indefinidas (10). Es dable tener en cuenta que los perros sin raza definida generalmente no obtienen muchos cuidados sanitarios por parte de sus propietarios, estando expuestos a situaciones que pueden favorecer la infección. Una vez producida, la atención veterinaria aún puede

Tabla 2. Razas de canes.

\begin{tabular}{lcc}
\hline raza & $\mathrm{N}^{\mathrm{o}}$ & $\%$ \\
\hline boxer & 1 & 5 \\
cocker spaniel & 1 & 5 \\
labrador retriever & 2 & 10 \\
sin raza definida & 10 & 50 \\
schnauzer & 4 & 20 \\
shihtzu & 2 & 10 \\
\hline total & 20 & 100 \\
\hline
\end{tabular}


demorarse, debido a la falta de condiciones económicas de los dueños.

Los resultados obtenidos indican que los aminoglucósidos, seguidos por las fluoroquinolonas, operan como eficaces opciones de tratamiento. Un uso racional de estos antibióticos se hace necesario para prolongar su utilidad en el tiempo, así como para investigar los aspectos moleculares y genéticos de la resistencia en $P$. aeruginosa.

Agradecimientos. A la Clínica Veterinaria La Colonial por el financiamiento y a la Lic. Maria G. Fagundes por su asistencia administrativa.

\section{REFERENCIAS}

1. Almeida MS et al. 2016. Isolamento microbiológico do canal auditivo de cães saudáveis e com otite externa na região metropolitana de Recife, Pernambuco. Pesquisa Vet Bras 36: 29-32.

2. Arais LR, Barbosa AV, Carvalho CA, Cerqueira AM. 2016. Antimicrobial resistance, integron carriage, and gyrA and gyrB mutations in Pseudomonas aeruginosa isolated from dogs with otitis externa and pyoderma in Brazil. Vet Dermatol 27: 113.

3. Bernal Y, Osorio K, Torres O. 2015. Pseudomonas aeruginosa: an emerging nosocomial trouble in veterinary. Rev MVZ Córdoba 20: 4937-4946.

4. Bugden DL. 2013. Identification and antibiotic susceptibility of bacterial isolates from dogs with otitis externa in Australia. Austr Vet J 91: 43-46.

5. Cardoso MJ et al. 2011. Dermatopatias em cães: revisão de 257 casos. Arch Vet Sci 16: 66-74.

6. Clinical and Laboratory Standards Institute - CLSI. 2013 Performance standards for antimicrobial disk and dilution susceptibility tests for bacteria isolated from animals. Public. Wayne P.A., Pennsylvania, 3rd ed., supplement VET01S.

7. de Oliveira VB et al. 2012. Etiologia, perfil de sensibilidade aos antimicrobianos e aspectos epidemiológicos na otite canina: estudo retrospectivo de 616 casos. Sem Ciênc Agr 33: 2367-2374.

8. Dégi J, Cristina RT, Stancu A. 2010. Otitis externa caused by bacteria of the genus Pseudomonas in dogs. Lucr Stiint Med Vet 43: 143-147.

9. Dégi J et al. 2013. Frequency of isolation and antibiotic resistance of staphylococcal flora from external otitis of dogs. Vet Rec 173: 42 .

10. Doublet B, Bousquet MA, Madec JY. 2012. Le concept «one health» en antibio résistance et les flux de genes. Innov Agron 24: 79-90.

11. Hariharan H, Coles M, Poole D, Lund L, Page R. 2006. Update on antimicrobial susceptibilities of bacterial isolates from canine and feline otitis externa. Canad Vet $J 47$ : 253-255.

12. Hariharan H, McPhee L, Heaney S, Bryenton J. 1995. Antimicrobial drug susceptibility of clinical isolates of Pseudomonas aeruginosa. Can Vet J 36: 166-168.
13. Ishii JB, Freitas JC, Arias MV. 2011. Resistência de bactérias isoladas de cães e gatos no Hospital Veterinário da Universidade Estadual de Londrina (2008-2009). Pesq Vet Bras 31: 533-537.

14. Korbelik J, Singh A, Rousseau J, Weese JS. 2018. Analysis of the otic mycobiota in dogs with otitis externa compared to healthy individuals. Vet Derm 29: 417.

15. Lin D et al. 2012. Characterization of antimicrobial resistance of Pseudomonas aeruginosa isolated from canine infections. J Appl Microbiol 113: 16-23.

16. Mekić S, Matanović K, Šeol B. 2011. Antimicrobial susceptibility of Pseudomonas aeruginosa isolates from dogs with otitis externa. Vet Rec 169: 125.

17. Morita Y, Tomida J, Kawamura Y. 2012. Primary mechanisms mediating aminoglycoside resistance in the multidrug-resistant Pseudomonas aeruginosa clinical isolate PA7. Microbiol 158: 1071-1083.

18. Paterson S. 2016. Discovering the causes of otitis externa. In Pract 38: 7-11.

19. Pedersen K et al. 2007. Occurrence of antimicrobial resistance in bacteria from diagnostic samples from dogs. $J$ Antimicr Chemother 60: 775-781.

20. Penna $\mathbf{B}$ et al. 2011. In vitro antimicrobial resistance of Pseudomonas aeruginosa isolated from canine otitis externa in Rio de Janeiro, Brazil. Braz J Microbiol 42: 14341436.

21. Petrov V et al. 2013. Otitis externa in dogs: microbiology and antimicrobial susceptibility. Rev Méd Vétér 164: 18-22.

22. Poole K. 2005. Aminoglycoside resistance in P. aeruginosa. Antimicrob Agents and Chemoth 49: 479-487.

23. Ragheb MN et al. 2019. Inhibiting the evolution of antibiotic resistance. Molec Cell 73: 157-175.

24. Robaj A, Sylejmani D, Hamidi A. 2015. Investigation and antimicrobial susceptibility of microbial agents of external otitis in dogs. J Anim Vet Adv 14: 277-280.

25. Schwarz $\mathbf{S}$ et al. 2010. Assessing the antimicrobial susceptibility of bacteria obtained from animals. J Antimicrob Chemother 65: 601-604.

26. Sfaciotte RA et al. 2015. Antimicrobial resistance in bacterial pathogens of canine otitis. Am J Anim \& Vet Sci 10: 162-169.

27. Shaw S. 2016. Pathogens in otitis externa: diagnostic techniques to identify secondary causes of ear disease. In Pract 38: 12-16.

28. Soler M, Tello M, Moreso JM, Riera L. 2000. Otitis externa en perros y gatos: aislamiento microbiológico y antibioterapia. Clín Vet Peq Anim 20: 72-75.

29. Türkyilmaz S. 2008. Antibiotic susceptibility patterns of Pseudomonas aeruginosa strains isolated from dogs with otitis externa. Turk J Vet \& Anim Sci 32: 37-42.

30. von Silva MS, Wolf G, Mueller RS. 2019. Determination of minimum inhibitory concentrations for silver sulfadiazine and other topical antimicrobial agents against strains of Pseudomonas aeruginosa isolated from canine otitis externa. Vet Dermatol 30: 145-e42. 